\title{
Research on recovery and utilization of Solid FAE
}

\author{
T.F. Shi \\ Ordnance Engineering College, Shijiazhuang, China \\ M.H. Chen \\ Ordnance Technology Research Institute, Shijiazhuang, China \\ Y.W. Fan \\ Jiangyang Company, Jinxi Industrial Group, Taiyuan 030041, China
}

\begin{abstract}
At present our army has a huge cloud explosion agent needs to be destroyed. The traditional incineration, landfill and other methods has not adapted to the current needs of the community. From view of recycling, through the discuss the recovery method of hexogen (RDX), ammonium perchlorate (AP) and aluminum (Al).The paper conclude a method of recovery and utilization of Solid FAE, by ultrasonic-water dissolve method and solvent extraction, to recovery the components of solid FAE. The scheme is feasible and effective in theory.
\end{abstract}

KEYWORD: Solid FAE; recovery; de-energizing; separation

\section{INTRODUCTION}

Solid FAE is also called thermobaric bomb, it can cause thermal effect and strong detonation overpressure after exposure, killing enemy live target exposed in complex terrain, buildings and unarmored vehicles. At the beginning of the last century, German started research on FAE .1966, the U.S. military in Vietnam War cast first FAE bomb, officially opened a competition in development of FAE (Guo Chengguang. 2003). With the weapons and equipment upgrading, powder charge in FAE change from liquid charge to solid loading. The solid FAE has uniform density, overcome the disadvantage of centroid shift occur in the process of transportation and storage (Wang Chenchen. 2011). PLA has decades of history of equipment solid FAE, there are a large number of abandoned solid FAE to deal with every year, it is urgent to find a safe and economical efficient methods to deal with it. Therefore, according to the existing circumstances in disposing the solid FAE, the method to deenergize abandoned solid FAE is studied. Research on this area is little, because components in passivation RDX-Al and solid propellant are similar with solid FAE, on the basis of the passivated RDX and solid propellant separation method, the method to separate solid FAE is researched.

\section{RECOVERY SOLID FAE COMPONENT}

Solid FAE have multiple components can be recycled, the study mainly to the recovery of hexogen (RDX),ammonium perchlorate (AP) and metal alu- minum (Al), RDX in solid FAE is about $40 \%$ of its weight, $\mathrm{AP}$ is about $13 \%, \mathrm{Al}$ is about $40 \%$. The other explosives contains the same ingredients can be well recycled in similar method, and can reach the standard as explosive material, also can be applied to the civilian application.

\section{$2.1 R D X$ recovery}

\subsubsection{Solvent extraction method}

The principle is: different components in the same solution have different solubility, only one constituent can dissolve a lot in the solvent, the other consists are slightly soluble or insoluble. After filtering can get slightly soluble or insoluble fractions, solution component can be extracted by recrystallization method (cool-crystallization, evaporationcrystallization, vacuum-cool- crystallization, precipitation-crystallization) (Wang Jun. 2012). In the 1970s, Cousins used solvent extraction methods and compared the effect of different solvent. Chen Yafang (Chen Yafang, 2012) also used this method to separate and recover TNT-RDX-Al explosive. The process flow process is shown in Figure 1.

\subsubsection{Water suspension method}

Because of containing compound wax and other substances, passivated RDX is not suitable for directly using solvent method to separate and recycle. Generally, water suspension method is effective. A lot of research has been done by many domestic manufacturers and research institutes, the process is shown in Figure 2. 


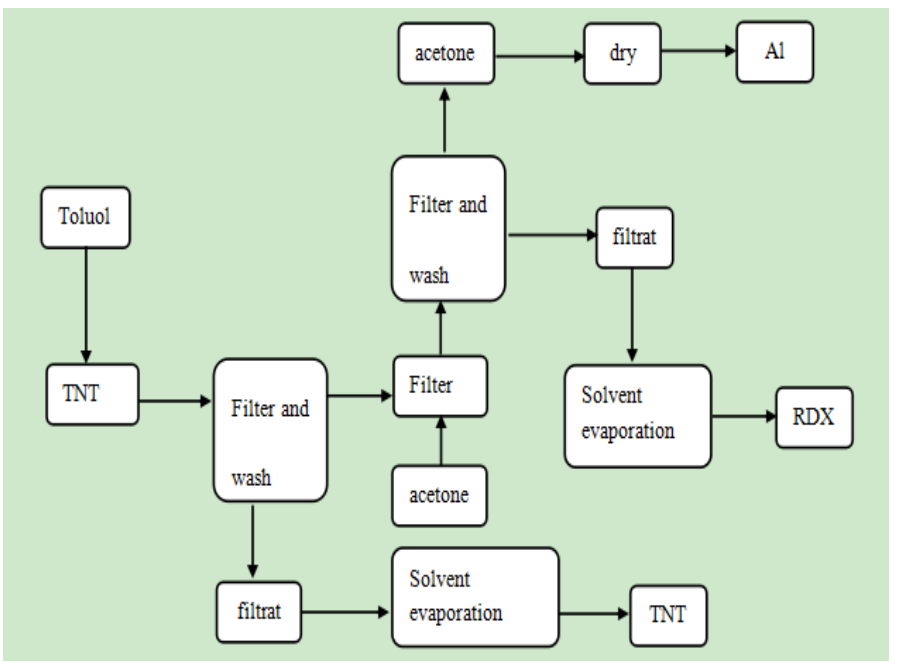

Fig. 1 the process flow chart of the separation of TNT-RDX-Al explosive

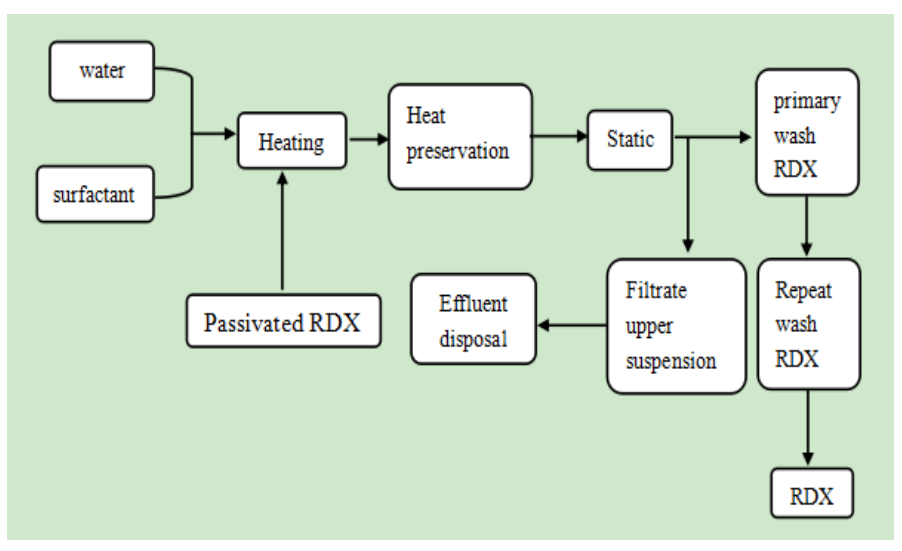

Fig. 2 the process flow chart of the water suspension method of passivated RDX

\subsection{Ammonium perchlorate $(A P)$ recovery}

Composite solid propellant containing a lot of AP, in general, aqueous extraction method and liquid ammonia extraction method are used to extract AP in composite solid propellant.

\subsubsection{Aqueous extraction}

Meldon J.Mcintosh extracted AP in aqueous extraction method.Graham C.Shaw reduced the surface viscosity and improved the efficiency of recycling by adding surface active agent. The AP solubility changes with temperature, which is the principle of aqueous extraction. The AP in explosive can be extracted through dissolving, filtration, recrystallization process. Application of this method is plenty in domestic.

\subsubsection{Ammonia extraction}

This method is based on compatible principle, because the polarity of AP and ammonia is similar, the solubility of AP in liquid ammonia increased greatly, for example, $t 25^{\circ} \mathrm{C}$, AP solubility in liquid ammonia can reach6 7 times compared in the water. In propellant, only AP can be dissolved in liquid ammonia, so this method can easily and quickly re- covery the AP. In order to achieve the purpose of extracting AP, the US army designed of highpressure anhydrous ammonia unit, the process is: the ammonia is injected into the propellant surface inside the engine to make the propellant into small particles, oxidant AP dissolve in liquid ammonia, and other insoluble components will be removed as sludge. Because of high cost, complicated condition, the research on this method is less.

\subsection{Aluminum (Al) recovery}

Aluminum is insoluble in common inorganic solvents and organic solvents, so its recovery is generally established on the basis of AP and RDX. Main methods are using organic solvent to remove organic impurities in residue by repeatedly washing and filtering .Robert A. Frosch used ethanol solution containing sodium methanol to initiate ester exchange reaction of the crosslinking binder system, the reaction product was dissolved in solvent.

\section{RECOVERY SCHEME OF SOLID FAE}

Because of the solid FAE contain rubber, compound wax and other substances, this paper selects ultrasonic-water dissolve method, rubber in solid FAE will be swelled, then the AP is dissolved in water, ultrasound can destroy grid structure, and facilitates separation. RDX and Al can be extracted by solvent extraction recovery method. The process is shown in Figure 3.

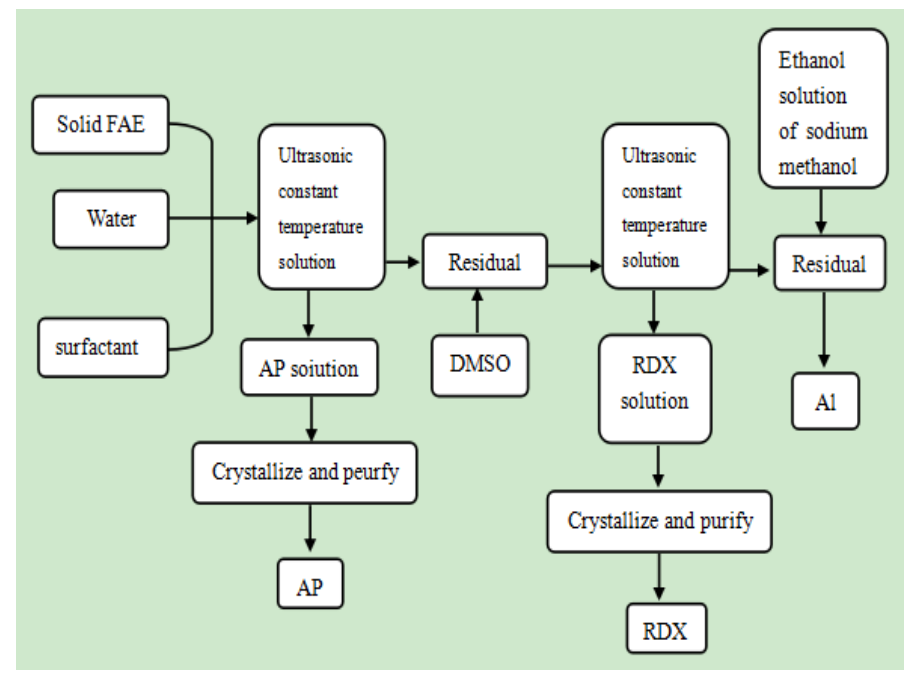

Fig. 3 separation process flow chart of solid cloud explosion

\section{CONCLUSION}

Abandoned solid FAE is dangerous in urgent need of disposing, traditional ammunition dispose method such incineration and deep burying method cause serious environmental pollution and enormous waste of resources. Recovery useful ingredients of solid 
FAE has great advantage in these two aspects. If this method is applied in industry, it will bring huge economic and environmental benefits.

\section{REFERENCE}

A.Frosch. Graham C. Shaw.Robert Recovery of aluminum from composite propellants: US.

Cousins, R.Hogge Edwin, C. A Comparison of Winona Extraction Solvent for the Analysis of Composition A-3 Explosive. AD-703217.

Chen Yafang, Wang Baoguo, Zhang Jinglin, et al. 2012. The recovery and characterization of RDX in the abandoned TNT-RDX-Al explosive. Chinese journal of explosives \&propellants, 32 (5): 23-25.

Guo Chengguang. 2003. Uncover the mystery of the "cloud explosion" . National defense technology foundation. 4344.

Jing Changlun, Xu Fuming. 2008. Study on the reuse of expired passivated RDX.Explosive materials, 37 (1):4-5.

Jiang Dayong, Wang Xuanjun, Li Bing, et al.2008. Research progress on the reuse of scrap HTPB. Initiator \& Pyrotechnics.35 (2):44-47.

Wang Chenchen. 2011. Study on the process and performance of solid cloud explosion. Nanjing: Nanjing University of cience and Technology.

Wang Jun. 2012. Study on leaching of ammonium perchlorate from composite solid propellant. Nanjing: Nanjing University of Science and Technology. 